\title{
Biomechanical Analysis of Uwan's Serve Technology for Elite Tennis Athlete
}

\author{
Xiangping Ye and Jihe Zhou \\ Chengdu Sport Institute, China
}

\begin{abstract}
In this paper, Using JVC model high-speed video camera for three-dimensional camera literature mathematical statistics and other research methods. Correcting the ITF Youth Masters women's single runner Uwan gets on biomechanical research. Using 3D Signal Tec analysis system for the shooting video recorded data analysis, to reveal the athletic characteristics of elite athletes serve technology and find out the model of elite athlete serve technique. For our tennis players to provide a solid reference data. This research indicates: In the toss ball stage, the athlete's main support leg has the smallest knee angle is $92.87^{\circ}$ at the end of the throwing stage. Uwan's kinematics parameter is 123.2 ${ }^{\circ}$. Uwan's shoulder joint angle gradually increased and elbow angle gradually smaller. In the swing back to the stage, Uwan's elbow flexion range larger and the racket swing distance longer. In the swing batting stage, her right shoulder, elbow angle and wrist joints angle, head speed in turn increased. With play stage, Uwan hit the ball more stringent package. This can be drawn: In the toss ball stage, Uwan's knee flexion is not enough and her center of gravity is high. In the swing back to the stage, Uwan swing a longer distance and well preparing for swinging forward. In the swing batting stage, Uwan's right shoulder angle slightly smaller than the world's best athletes and there is still much room for improvement. With play stage, Uwan's legs stretch without the best athletes in the world.
\end{abstract}

\section{Keywords—tennis; serve; biomechanical analysis}

\section{RESEARCH PURPOSES}

In this paper, through the serve technique getting on the three-dimensional video game technology analysis and analysis and discussion of the signification of the key links of the service technology. Hope to find out the best tennis player serve technical mode and demonstrate the Sport Biomechanics of Tennis Serve Technology for our tennis player to provide some reference data.

\section{RESEARCH OBJECT AND METHOD}

\section{A. Research Object}

The Slovenian tennis player KaJia-Uwan and 2017 ITF Tennis Masters singles runner-up.

\section{B. Research Method}

Video analytic method: In the Chengdu International Tennis Exchange Center Youth Tennis Masters solid three-dimensional shooting excellent tennis player KaJia.Uwan's serve technology. Adopting the 3DSignalTec system developed by Beijing Senmiao Xin Company to conduct data analysis on the shooting video shot. Analysis of the selection of European Dempster mannequin. According to research needs, analytical added shoulder hip joint projection angle, tennis racket top and tennis three test points. Digital video capture speed of 50 frames / second. Using simple video analysis. Sampling speed of $100 /$ second. Sampling time is5 seconds. The resulting data is smoothed using low-pass filtering. Computer data output frequency is $50 \mathrm{~Hz}$. The cut-off frequency is $8 \mathrm{~Hz}$.

\section{Documentation method: Check the relevant literature}

Data Statistics: The results of video analysis of data statistics and finishing

\section{RESEARCH RESULT}

\section{A. Analysis of the Ball Throwing Stage}

1) Technical action essentials: Throwing ball stage is the ball and hand separation time to the highest point of the ball's moment. The essentials of its action is to throw the ball to be stable, the station is good, the body naturally relaxed.

2) Parameter analysis: At this stage, through the video playback can be drawn: Uwan with the shoulder joint as the fulcrum, the arm down the ball to the ball vertically in the process of throwing. Uwan at the moment of separation of the ball and hand right shoulder and right elbow angle are $52.0^{\circ}$. $153.3^{\circ}$. At the highest point of the ball, the right shoulder and right elbow angles are $89.6^{\circ}$ and $67.0^{\circ}$. The angle of shoulder gradually increased and the angle of the elbow gradually reduced. The throwing height is 3.624 meters. The maximum height of the ball up to 3.88 meters. Uwan's throwing height is higher than Moya's throwing height. So she is more difficult to grasp the batting point. During the throwing stage, the athlete is required to exercise full squat. This action can contract joints and muscles. Then effectively drive the ankle, knee and hip movement [1].The moment of maximum knee flexion, Uwan's shoulder angle of projection of $18.7^{\circ}$. Her left knee joint angle was $133.2^{\circ}$ and right knee joint angle was $123.2^{\circ}$. At the end of this period of Uwan, The minimum knee angle reached was 123.2.Athletes at the end of throwing the main support leg knee angle is the smallest $92.87^{\circ}$ [2]. This can be drawn: Her knee flexion is not enough, turning radius is small, is not conducive to lower extremity stretch at the end of the toss.

\section{B. Backward Swing Phase Analysis}

1) Technical action essentials: The swinging stage is the process of dropping the ball to the highest point to the moment of the lowest point of the racket. The essentials of its action is: 
the formation of anti-bow, lower leg pedal stretching action fully.

2) Parameter analysis: The entire serve is a coordination chain from the biomechanical point of view of the service process. Her left and right knee angles were $174.5^{\circ}$ and $158.8^{\circ}$ When the racket behind the lowest moment. Swing backward for 0.2 seconds. The minimum elbow angle is $66.4^{\circ}$. Commonly known as "scratch back"[3]. From the racket shoulder point of view, holding racquet shoulder angle increases can not only increase the rate of "scratch back" action, but also make shoulder joint storage more elastic potential energy. The posterior knee flexion range of about $45.2^{\circ}$ and $37.3^{\circ}$. Her knee flexion maximum angular velocity of $324.8 \%$ s. At this stage, Uwan swing a longer distance. This can increase the swing distance and well prepared for the forward swing.

\section{Swing Batting Stage Analysis}

1) Technical action essentials: Swing batting stage is the lowest point of the racket to touch the ball moment of the process. The essentials are: hit the high point, a complete whipping action.

2) Parameter analysis: Maintaining the swing angle of the shoulder joint at the end of the backward swinging stage can increase the turning radius of the last joint around the shoulder joint and increase the corresponding linear speed so as to enhance the effect of the "whipping" action in the swing batting stage. The world's top tennis players Moya and Aino's Shoulder angles are $135.39{ }^{\circ}$ and $139.36^{\circ}$. Uwan right shoulder joint is $135.3^{\circ}$ at this time. Her shoulder is slightly smaller than the world's best athletes. Uwan's right shoulder, elbow and wrist speed were $2.97 \mathrm{~m} / \mathrm{s}, 4.66 \mathrm{~m} / \mathrm{s}, 8.53 \mathrm{~m} / \mathrm{s}$, head speed is $21.03 \mathrm{~m} / \mathrm{s}$. Her shoulder, elbow wrist head speed in turn increasing, so indicating her swing action in line with "whipping" principle. In the touch the ball moment, the ball height of 1.943 meters, the ball speed of $39.47 \mathrm{~m} / \mathrm{s}$. Uwan's throw height and hit the difference between the heights of 1.681 meters.

\section{With the Stage of Play Analysis}

1) Technical action essentials: With the play phase is the time to touch the ball to the left foot toe moment to the process. The essentials are: swing arm with the natural completion of the completion of the lower extremity buffer.

2) Parameter analysis: Swing arm naturally complete with the wave, the lower limbs to complete the buffer, rhythm smooth, in line with the biomechanical consistency. Uwan at the left foot toe at a moment when the left knee joint angle of $152.5^{\circ}$, right knee joint angle of $142.3^{\circ}$. The kinematic parameters of the world elite men tennis player Dimitrov were $162.4^{\circ}$ and $165.5^{\circ}$ [4]. This shows Youwan legs stretch without the best athletes in the world.

\section{ANALYSIS CONCLUSION}

Through the above analysis, we can draw the following conclusions:
In the throwing ball stage: Uwan throw a little high (3.624 meters) and Uwan's knee flexion and extension angles were $92.87^{\circ}, 123.2^{\circ}$ in the maximum moment of knee flexion. You Wan knee is not full, high center of gravity.

In the swing back to the stage: The posterior knee flexion range of about $45.2^{\circ}$ and $37.3^{\circ}$. At this stage, Uwan swing a longer distance. This can increase the swing distance and well prepared for the forward swing.

In the swing batting stage: Uwan right shoulder angle of $135.3^{\circ}$ and slightly smaller than the world's best athletes. There is a lot of room for improvement. Her right shoulder, elbow and wrist joint speed were $2.97 \mathrm{~m} / \mathrm{s}, 4.66 \mathrm{~m} / \mathrm{s} 、 8.53 \mathrm{~m} / \mathrm{s}$, head speed of $21.03 \mathrm{~m} / \mathrm{s}$, her swing action in line with the "whipping" principle.

With play stage: Uwan's legs stretch without the best athletes in the world.

\section{REFERENCES}

[1] Guo Jun .Kinematics Analysis of the Serving Techniques of the World Elite Female Tennis Players [D]. Chengdu Sport University, 2016.

[2] Kou Jiaqi. Moya and Ainyu tennis serve technology comparative analysis [A]. Chinese sports science society sports biomechanical branch. Sixteenth national sports biomechanics academic exchange conference (CABS 2013) [C]. China Sports Science Society Sports Biomechanics: 2013: 2 .

[3] Advances in Computer Simulation of Digitized 3D Human Motion [J]. Chinese Academy of Sciences, 2004 (03): 206-207 + 239.

[4] DU Chuan jia. Dynamic Analysis of Three-dimensional Virtual Reproduction of Elite Tennis Player Dimitrov Serve [A] .China Sports Science Institute .2015 Tenth National Sports Science Conference Proceedings Compilation (B) [C]. China Sports Science Institute, 2015: 3. 